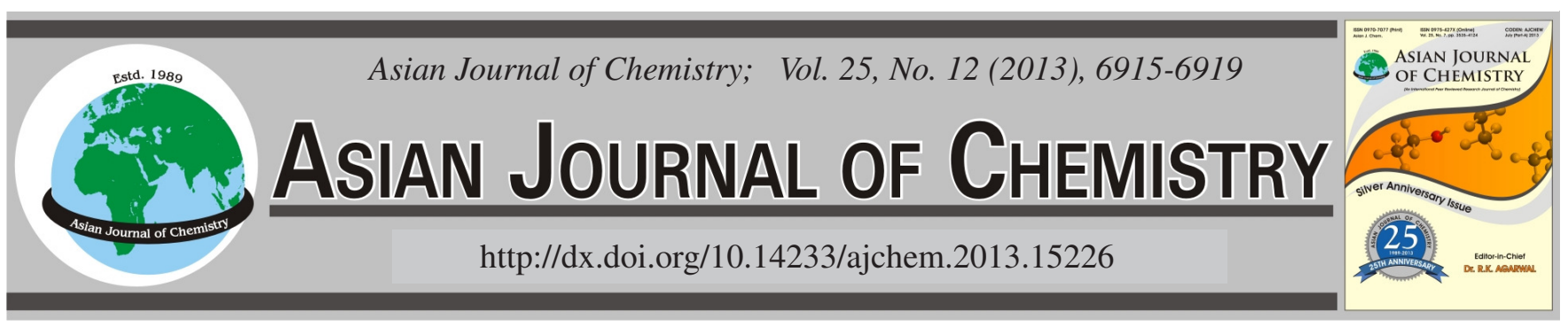

\title{
Effect of Solution Factors on Endotoxin Coagulation State
}

\author{
Hemin Li ${ }^{1}$, Cunyu Li ${ }^{1}$, Yunfeng Zheng ${ }^{1}$, Xinglei Zhi ${ }^{1}$, Guoping Peng ${ }^{1, *}$, Helai Li $^{2}$ and Hongbo Lu ${ }^{3}$
}

${ }^{1}$ School of Pharmacy of Nanjing University of Chinese Medicine, Nanjing University of Chinese Medicine, Nanjing City 210046, P.R. China ${ }^{2}$ Unit No. 61769 of PLA, Shanxi Wenshui City 041000, P.R. China

${ }^{3}$ Hot Rolling Plant of Meishan Iron Steel Co., Nanjing City 210039, P.R. China

*Corresponding author: Tel./Fax: +86 25 86798186; E-mail: guopingpeng@ sohu.com

\begin{abstract}
Endotoxin contamination of drugs intended for parenteral use presents a serious problem. Endotoxin removal efficiency differs under various conditions (temperature, $\mathrm{pH}$ and solvent). This variation can be related to the endotoxin agglomerate state. There is no direct way to probe endotoxin agglomerate state. In this study, the endotoxin agglomerate size was measured directly by a nanoparticle-size instrument and endotoxin removal efficiency was indirectly analyzed under the same conditions with ultrafiltration technology. Direct-indirect analysis of endotoxin agglomerate state was established. We investigated the mechanisms of this interference and found that temperature, $\mathrm{pH}$ and ethanol exerted significant effects on endotoxin removal from water solutions and endotoxin coagulation size. Endotoxin removal efficiency and endotoxin agglomerate size were determined under varying temperatures, $\mathrm{pH}$ levels, and ethanol concentrations by ultrafiltration and by using an endotoxin nanometer particle size analyzer. When the temperature was increased from 10 to $60{ }^{\circ} \mathrm{C}$, the endotoxin agglomerate size and endotoxin removal efficiency increased. When $\mathrm{pH}$ was increased from 3 to 10 and ethanol concentration raised from 10 to $30 \%$, the endotoxin agglomerate size and the endotoxin removal efficiency decreased. This study provides reference data for endotoxin removal and improves injection safety.
\end{abstract}

Key Words: Endotoxin, Agglomerate state, Particle size distribution, Temperature, pH, Ethanol.

\section{INTRODUCTION}

Endotoxins are the outer cell membrane constituents of Gram-negative bacteria. Serious endotoxin intoxication can cause sepsis and septic shock, leading to severe hypertension, cardiovascular collapse, multiple organ failure and death ${ }^{1}$. Bacterial endotoxins are recognized as a major cause of pyrogenic reactions that can occur during administration of traditional Chinese medicine ${ }^{2}$. However, the endotoxin removal efficiency (ERE) from different injections fluctuates with the same molecular weight cut off membrane. This variation in endotoxin removal efficiency occurs because the endotoxin agglomerate state differs in various solutions and environments. However, no accurate and simple method has been developed to analyze the endotoxin molecular state in solutions. Numerous methods such as ion exchange adsorption ${ }^{3}$, ion exchange membrane ${ }^{4}$, ion-exchange filter ${ }^{2}$, ultrafiltration ${ }^{5,6}$, extraction ${ }^{7}$ and activated carbon ${ }^{8}$ have been studied to remove endotoxin, indirectly reflecting the molecular state in solutions. However, endotoxin exhibits various surfactant properties because its molecule is composed of a hydrophilic polysaccharide moiety, which is covalently linked to a hydrophobic lipid moiety ${ }^{9}$. The endotoxin agglomerate state is also influenced by temperature, $\mathrm{pH}$ and organic solvent. Therefore, the aforementioned techniques have several disadvantages in analyzing the endotoxin state.

To determine the endotoxin state, factors such as temperature, $\mathrm{pH}$ and ethanol were chosen to analyze the agglomerate laws. In this study, the endotoxin agglomerate size was directly determined by a nanometer-particle size analyzer, whereas the endotoxin removal efficiency was determined by ultrafiltration, which could only indirectly determine the endotoxin agglomerate state. The two methods mutually confirmed the endotoxin agglomerate state. This study can provide reference data for endotoxin removal from the injections and improve injection safety.

\section{EXPERIMENTAL}

Endotoxin standard substances were purchased from the National Institute for the Control of Pharmaceutical and Biological Products in China (Lot. No. 150601-201070). Limulus amebocyte lysate (LAL) was obtained from Zhanjiang Bokang Ocean Creature Co., Ltd. in China (Lot. No. 1003090, $\lambda=0.03 \mathrm{EU} / \mathrm{mL}$ ). All solutions were prepared with endotoxinfree water (LAL reagent water). 
Labware: The endotoxin size distribution was measured with Malvern ZEW3600 Zetasizer Nano System (Malvern Instruments). Two spiral wound membranes (Millipore Co., USA; Cat. No. CDUF006TQ) were used as the filter media. An endotoxin detector (Tianda Tianfa Technology Co. Ltd., China; Mode: BET-16M) was used to detect endotoxin concentration.

Pyrogen-free water was obtained from a Milli-Q ultrafiltration system (Millipore, Bedford, MA, USA). All glassware used were treated with $2 \mathrm{M} \mathrm{KOH}$ containing $30 \%$ ethanol and then heated in an oven at $250^{\circ} \mathrm{C}$ for $2 \mathrm{~h}$ and pyrogen-free water. The glassware were then heat-treated at $180^{\circ} \mathrm{C}$ overnight. All solution transfers were performed with endotoxin-free devices. Sterile disposable plasticware was used at all times to prevent endotoxin contamination.

Filtration: Endotoxin adsorption on membranes was examined using 50 and $100 \mathrm{kDa}$ Millipore membranes, which were washed with $0.1 \mathrm{M} \mathrm{NaOH}$ and pyrogen-free water to ensure absence of endotoxins on the membrane ${ }^{10}$. After equilibration with a $1500 \mathrm{~mL} 20 \mathrm{mM}$ phosphate buffer, a $1500 \mathrm{~mL}$ water solution containing approximately $100 \mathrm{EU} / \mathrm{mL}$ was filtered through the membrane at a flow rate of $30 \mathrm{~mL} / \mathrm{min}$. This flow rate was chosen because no alteration of the adsorption behaviour was evident compared with lower flow rates ${ }^{9,11}$. The endotoxin concentrations of the filtrates were determined by LAL test. The endotoxin removal ability of the process was quantitatively evaluated using endotoxin removal efficiency, which was determined by the following equation:

$$
\text { Endotoxin removal efficiency }(\%)=\frac{\mathrm{C}_{\mathrm{f}}-\mathrm{C}_{\mathrm{p}}}{\mathrm{C}_{\mathrm{f}}} \times 100 \%
$$

where $\mathrm{C}_{\mathrm{f}}$ is the endotoxin concentration (EU) in the stock solution and $\mathrm{C}_{\mathrm{p}}$ is the endotoxin concentration (EU) in the filtrate solution. Samples were frozen to prevent microbial growth if not measured immediately after the experiment. All test samples were mixed with an ultrasonic washer for $10 \mathrm{~min}$ before the samples were determined.

A specific volume of pyrogen-free water was mixed with a specific volume of endotoxin stock to reach a final endotoxin concentration of about $100 \mathrm{EU} / \mathrm{mL}$. The endotoxin solutions were filtered by 50 and $100 \mathrm{kDa}$ Millipore membranes, respectively, at $10,20,30,40,50$ and $60{ }^{\circ} \mathrm{C}$. The endotoxin concentration in filtrates was determined when the adsorption of membranes was saturated. The ultrafiltration temperature was controlled by water-bath water or ice to ensure that the whole solution temperature was constant. All feeds applied to ultrafiltration in the study of temperature effect are summarized in Table-1.

TABLE-1

SUMMARY OF FEEDS APPLIED TO ULTRAFILTRATION AND MALVERN LASER PARTICLE ANALYZER IN THE STUDY OF THE TEMPERATURE EFFECT

\begin{tabular}{ccc}
\hline Cases & Feeds & Temperature $\left({ }^{\circ} \mathrm{C}\right)$ \\
\hline 1.1 & Endotoxin solution & 10 \\
1.2 & Endotoxin solution & 20 \\
1.3 & Endotoxin solution & 30 \\
1.4 & Endotoxin solution & 40 \\
1.5 & Endotoxin solution & 50 \\
1.6 & Endotoxin solution & 60 \\
\hline
\end{tabular}

To study the effect of $\mathrm{pH}$ on endotoxin concentration, an endotoxin water solution was prepared with buffers at various pH levels: 3, 4.5, 6, 7, 8, 9 and 10. The buffers used were acetate buffer ( $\mathrm{pH}$ at 3 and 4.5), phosphate buffer ( $\mathrm{pH}$ at 6, 7, and 8), Tris- $\mathrm{HCl}$ buffer ( $\mathrm{pH} 9)$ and ammonia.ammonium chloride buffer ( $\mathrm{pH} 10)$. The concentration was $0.05 \mathrm{M}$ for all buffer solutions. First, pyrogen-free water and endotoxin were prepared separately with the same buffer at a specific $\mathrm{pH}$. A certain volume of pyrogen-free water was then mixed with a certain volume of endotoxin stock to obtain a final endotoxin concentration of about $100 \mathrm{EU} / \mathrm{mL}$. The endotoxin solution was filtered using 50 and $100 \mathrm{kDa}$ Millipore membranes, respectively. All feeds used to study the effect of $\mathrm{pH}$ are listed in Table-2 as cases 2.1, 2.2, 2.3, 2.4 and 2.5.

TABLE-2

SUMMARY OF FEEDS APPLIED TO ULTRAFILTRATION AND MALVERN LASER PARTICLE ANALYZER TO STUDY THE PH EFFECT

\begin{tabular}{cl}
\hline Cases & \multicolumn{1}{c}{ Feeds } \\
\hline 2.1 & Endotoxin solution with 0.05 M acetate buffer (pH 3) \\
2.2 & Endotoxin solution with 0.05 M acetate buffer (pH 4.5) \\
2.3 & Endotoxin solution with 0.05 M phosphate buffer (pH 6) \\
2.4 & Endotoxin solution with 0.05 M phosphate buffer (pH 7) \\
2.5 & Endotoxin solution with 0.05 M phosphate buffer (pH 8) \\
2.6 & Endotoxin solution with 0.05 M Tris-HCl buffer (pH 9) \\
2.7 & Endotoxin solution with 0.05 M ammonia-ammonium \\
& chloride buffer (pH 10) \\
\hline
\end{tabular}

Pyrogen-free water prepared with a certain volume of ethanol was mixed with endotoxin to reach a final endotoxin concentration of about $100 \mathrm{EU} / \mathrm{mL}$ and ethanol concentrations of $10,15,20$ and $30 \%$. The mixtures were filtered using 50 and $100 \mathrm{kDa}$ Millipore membranes, respectively. All feeds for the study of the ethanol effect are listed in Table-3.

TABLE-3

SUMMARY OF FEEDS APPLIED TO ULTRAFILTRATION AND MALVERN LASER PARTICLE ANALYZER IN THE STUDY OF ETHANOL EFFECT

Cases Feeds

$3.1 \quad$ Endotoxin solution with $10 \%$ ethanol concentration

3.2 Endotoxin solution with $15 \%$ ethanol concentration

3.3 Endotoxin solution with $20 \%$ ethanol concentration

$3.4 \quad$ Endotoxin solution with $30 \%$ ethanol concentration

Endotoxin assay: To determine the endotoxin concentration, LAL test was used ${ }^{12}$. All samples were measured in duplicate. The sensitivity of this test was $0.01 \mathrm{EU} / \mathrm{mL}$. The reaction mixtures were analyzed by endotoxin detection instrument, and results were compared with a standard curve to obtain endotoxin concentrations.

Measurements of endotoxin particle size distribution: A Malvern laser particle analyzer combined with the dynamic light scattering theory, measured Brownian motion to determine the endotoxin size distribution in the feeds. Brownian motion exhibited a random particle movement and was affected by temperature fluctuation and dispersant, among others. The laser source wavelength was $633 \mathrm{~nm}$, and the water refractive index was 1.330. All test samples were mixed with ultrasonic washer for $10 \mathrm{~min}$ before they were determined. 


\section{RESULTS AND DISCUSSION}

Endotoxin removal efficiency at varying temperatures: The endotoxin concentrations in the feeds and the filtrate with 50 and $100 \mathrm{kDa}$ Millipore membranes are shown in Figs. 1 and 2 , respectively, for cases $1.1,1.2,1.3,1.4,1.5$, and 1.6, in which the feeds were prepared at varying temperatures. Endotoxin removal efficiency is shown in Fig. 3 as a function of temperature. As indicated in Fig. 3, endotoxin removal efficiency increases significantly from 16 to $56 \%$ and from 9 to $39 \%$ by 50 and $100 \mathrm{kDa}$ Millipore membranes, respectively, when temperature increases from 10 to $60{ }^{\circ} \mathrm{C}$. By comparing the endotoxin removal efficiency in the two aforementioned situations, i.e., feeds filtered by 50 and $100 \mathrm{kDa}$ Millipore membranes, the changes in endotoxin removal efficiency versus temperature followed a similar pattern.

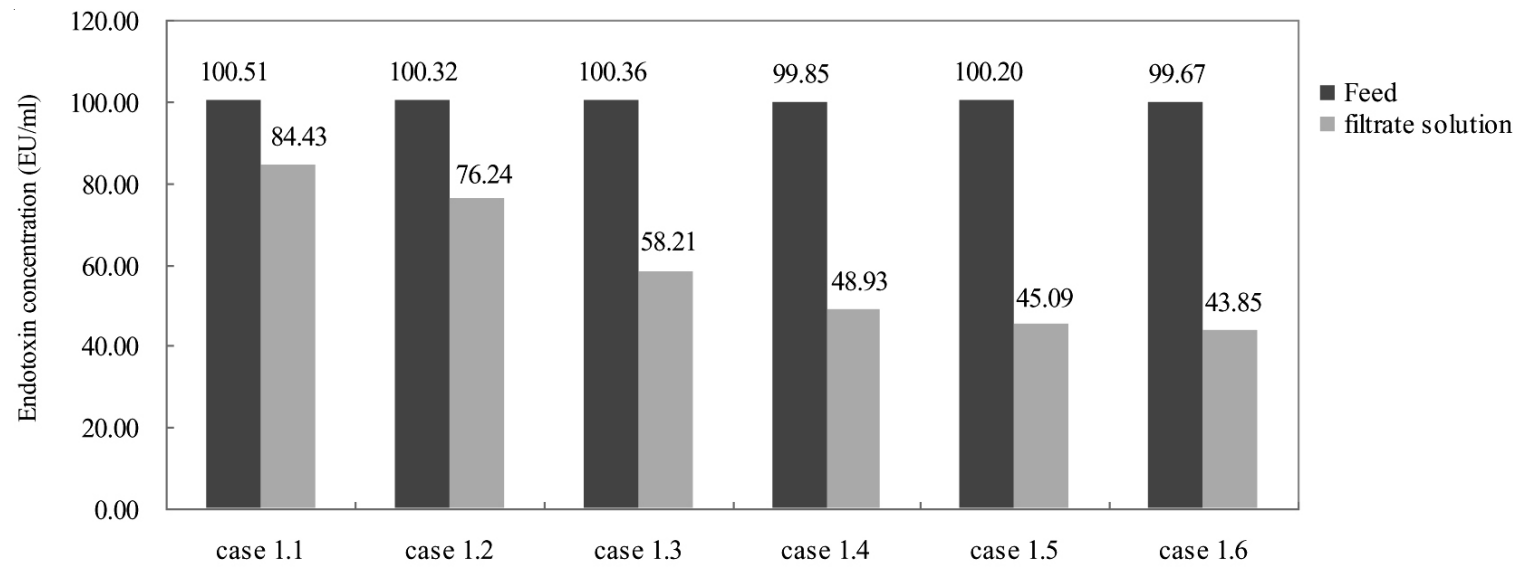

Fig. 1. Endotoxin removal from the endotoxin solution at varying temperatures by a $50 \mathrm{kDa}$ Millipore membrane

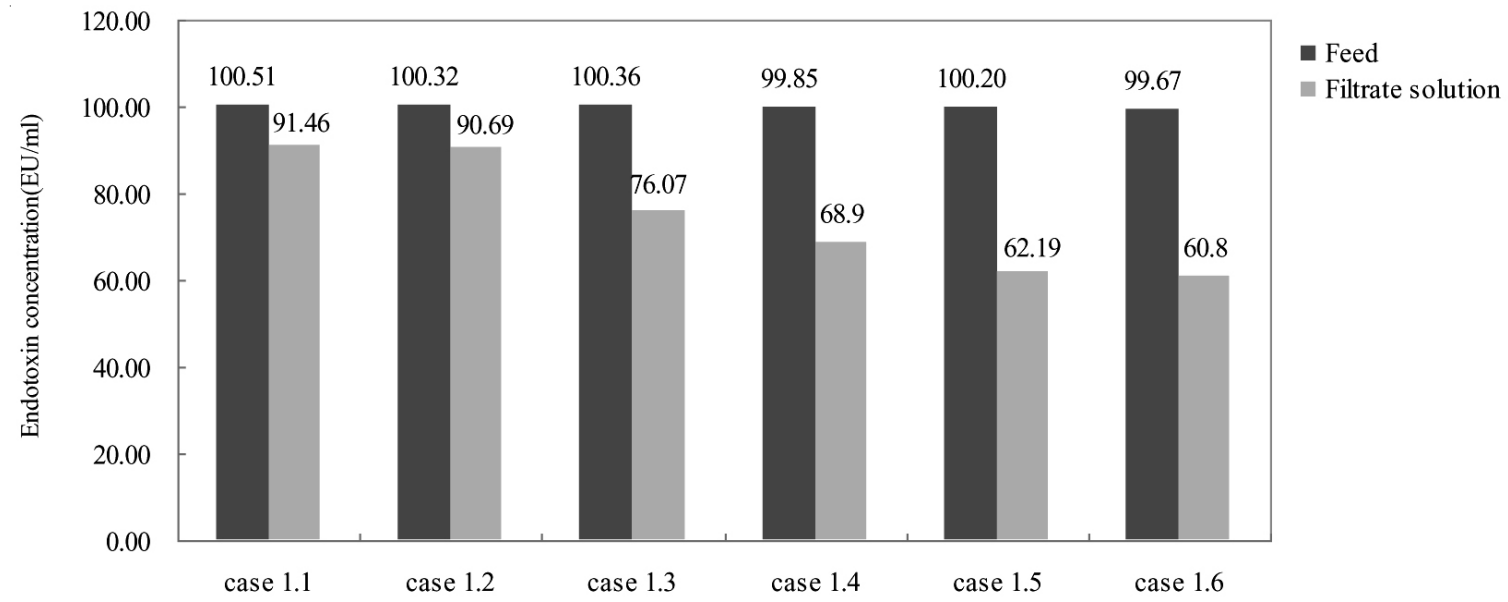

Fig. 2. Endotoxin removal from the endotoxin solution at varying temperatures by a $100 \mathrm{kDa}$ Millipore membrane

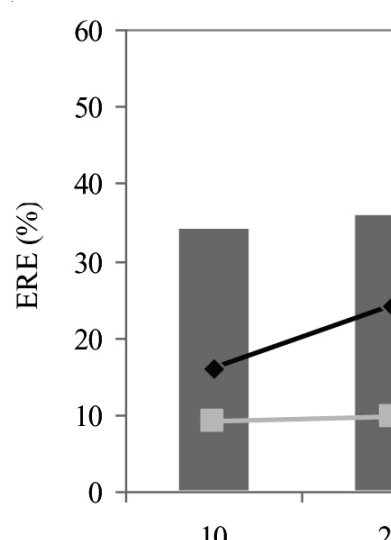

20

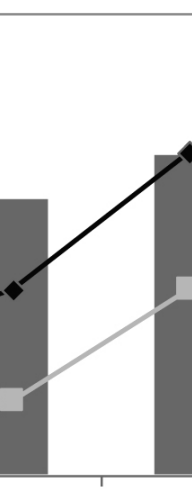

30

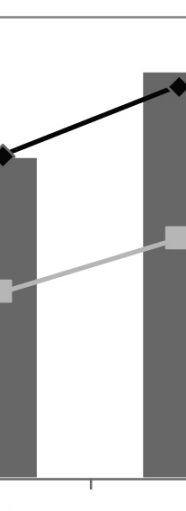

40

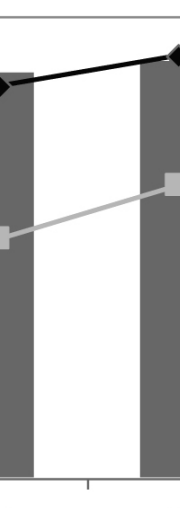

${ }^{\circ} \mathrm{C}$

Fig. 3. Effect of temperature on endotoxin removal efficiency and endotoxin size distribution by 50 and $100 \mathrm{kDa}$ Millipore membranes, respectively 
Endotoxin size distribution at varying temperatures: The size distribution of about $100 \mathrm{EU} / \mathrm{mL}$ water solution is shown in Fig. 3 for cases 1.1, 1.2, 1.3, 1.4, 1.5, and 1.6. The endotoxin size distribution was $511.7 \mathrm{~nm}$ at $10{ }^{\circ} \mathrm{C}$. As temperature increased from 10 to $50{ }^{\circ} \mathrm{C}$, the endotoxin size distribution increased significantly from 511.7 to $812.8 \mathrm{~nm}$. When the temperature increased from 50 to $60^{\circ} \mathrm{C}$, the endotoxin size distribution remained almost constant at a level of about $815.1 \mathrm{~nm}$. The endotoxin size distribution and the endotoxin removal efficiency exhibited a consistent tendency to change as the temperature increased.
Effect of pH on endotoxin removal efficiency: Endotoxin solution mixtures prepared with buffers of various $\mathrm{pH}$ levels were applied to 50 and $100 \mathrm{kDa}$ Millipore membranes, respectively. The effects of $\mathrm{pH}$ variation on the endotoxin concentration are presented in Figs. 4 and 5. The endotoxin removal efficiency is shown in Fig. 6 correspondingly. As $\mathrm{pH}$ increased from 3.0 to 10.0 , endotoxin removal efficiency decreased gradually from 50 to $20 \%$ and from 28 to $8.3 \%$ by 50 and $100 \mathrm{kDa}$ Millipore membranes, respectively. The figures indicate that the endotoxin removal efficiency generally decreased with the increase in $\mathrm{pH}$.

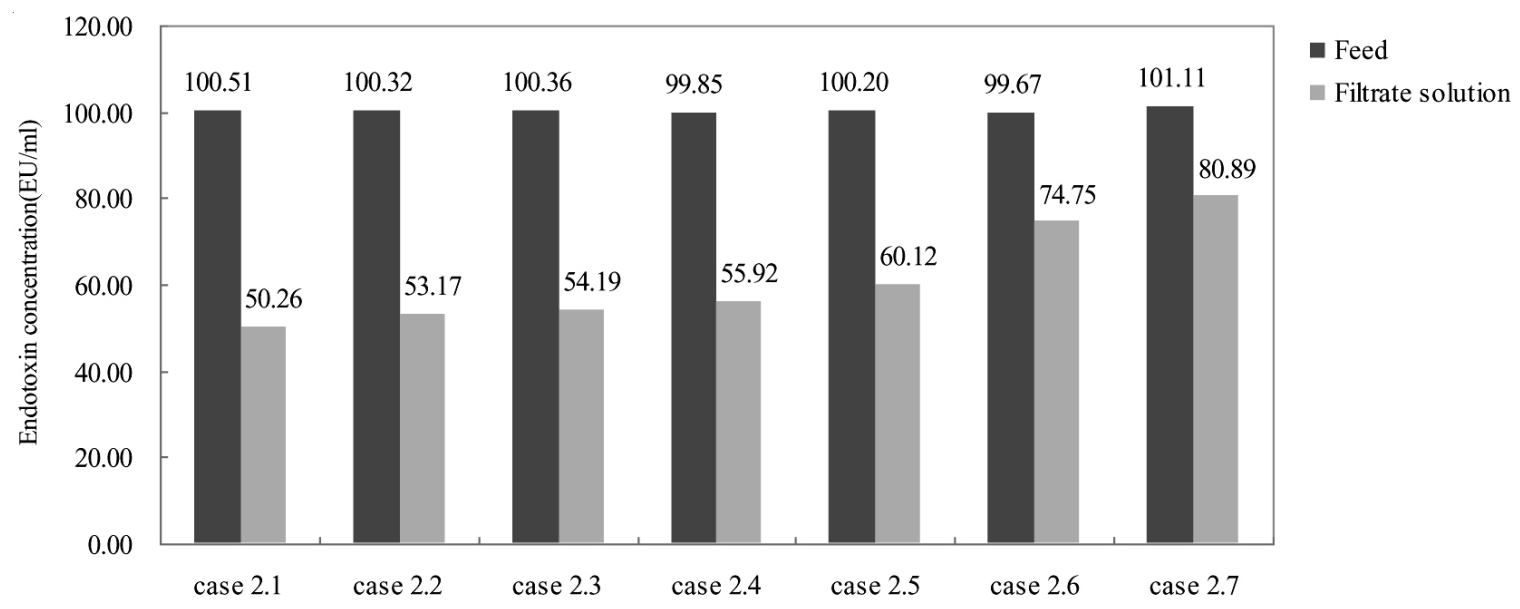

Fig. 4. Endotoxin removal from water solution at varying $\mathrm{pH}$ levels by a $50 \mathrm{kDa}$ Millipore membrane

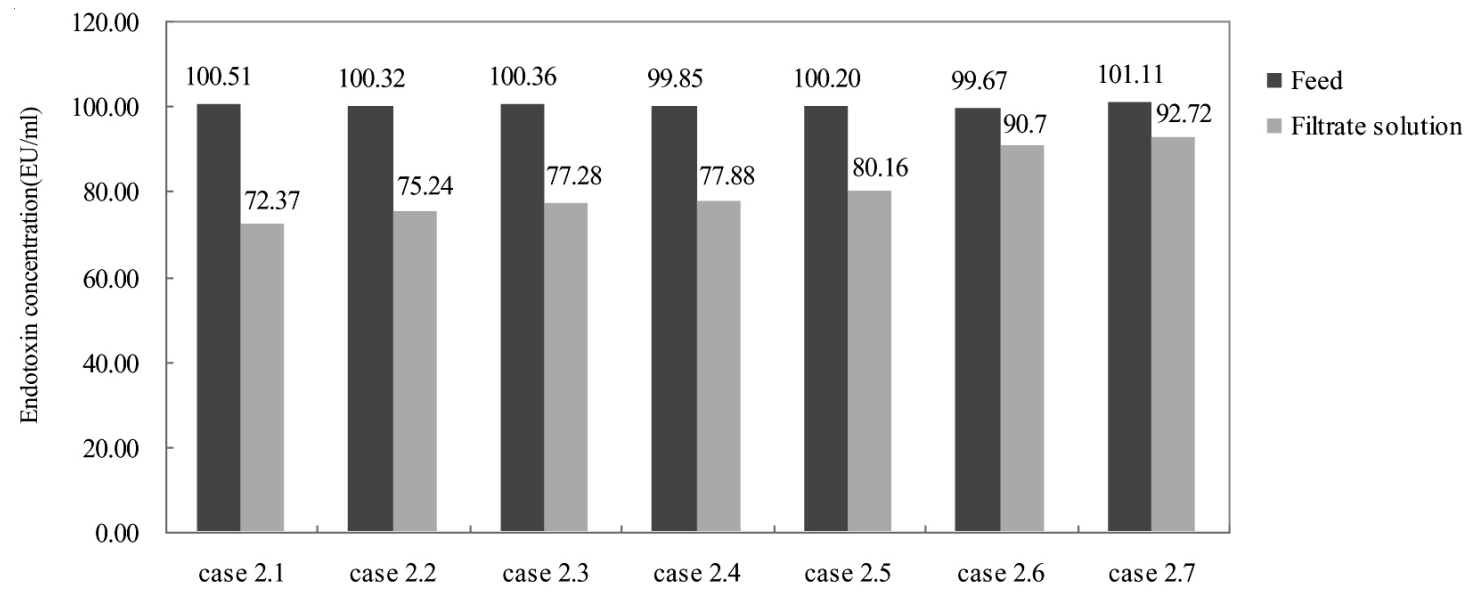

Fig. 5. Endotoxin removal from water solution at various $\mathrm{pH}$ levels by $100 \mathrm{kDa}$ Millipore membrane

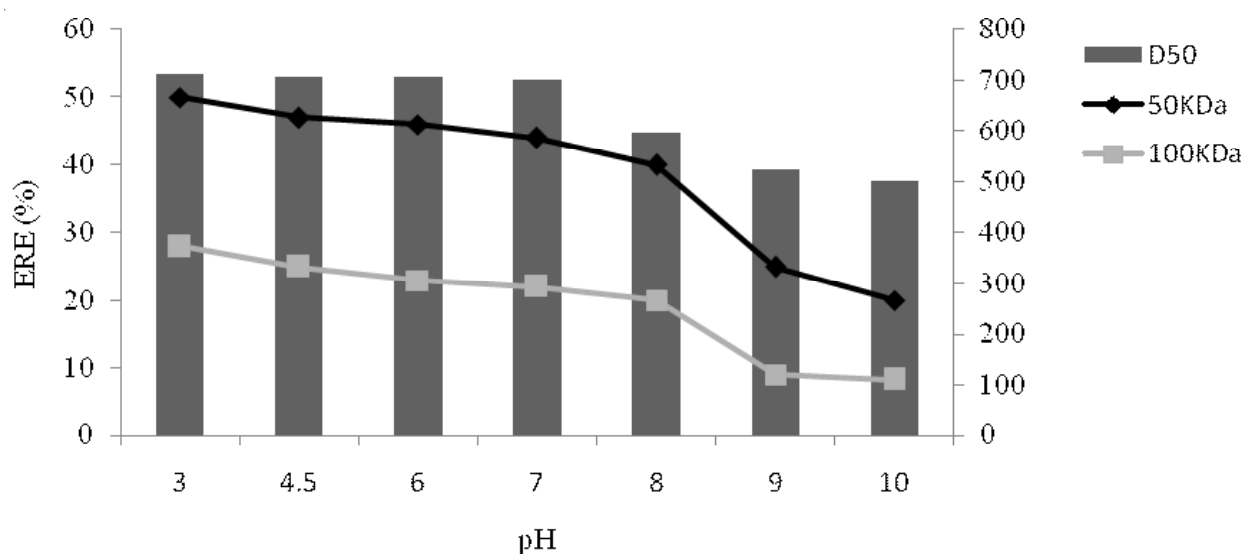

Fig. 6. Effect of $\mathrm{pH}$ on endotoxin removal efficiency and endotoxin size distribution by 50 and $100 \mathrm{kDa}$ Millipore membranes, respectively 


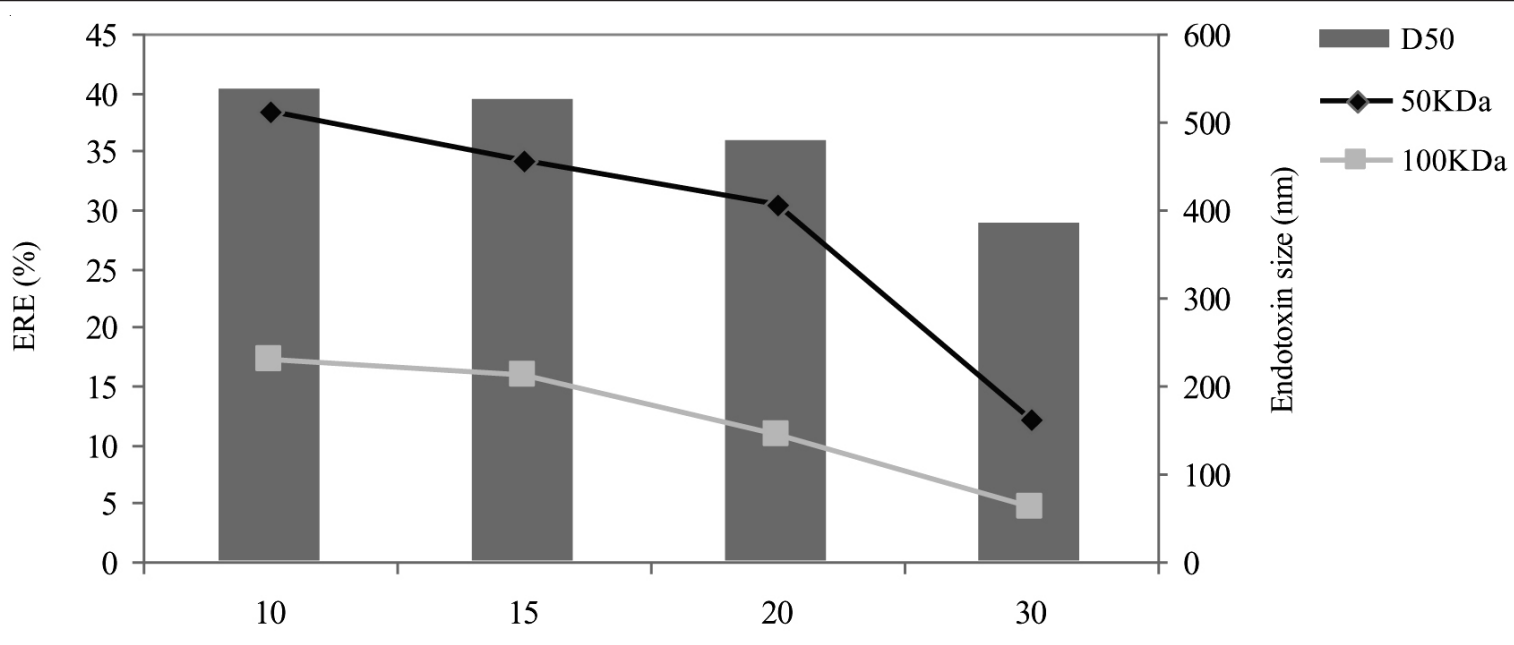

Ethanol concentration (\%)

Fig. 7. Effect of ethanol on endotoxin removal efficiency and endotoxin size distribution by 50 and $100 \mathrm{kDa}$ Millipore membranes, respectively

Effect of pH on endotoxin size distribution: The size distribution of about $100 \mathrm{EU} / \mathrm{mL}$ water solution prepared with buffers at varying $\mathrm{pH}$ levels is shown in Fig. 6 for cases 2.1, 2.2, 2.3, 2.4, 2.5, 2.6, and 2.7. As $\mathrm{pH}$ increased from 3.0 to 10.0, the endotoxin size distribution decreased gradually from 713.5 to $500.5 \mathrm{~nm}$. The change in endotoxin size distribution was consistent with the endotoxin removal efficiency.

Effect of ethanol on endotoxin removal efficiency: Approximately $100 \mathrm{EU} / \mathrm{mL}$ water solution mixtures prepared with ethanol to $10,15,20$ and $30 \%$ ethanol concentrations were applied to 50 and $100 \mathrm{kDa}$ Millipore membranes for cases 3.1, 3.2, 3.3 and 3.4. Data from Fig. 7 indicate that the endotoxin removal efficiency decreased gradually from 58.4 to $22.1 \%$ and from 27.2 to $9.6 \%$ by 50 and $100 \mathrm{kDa}$ Millipore membranes, respectively, as ethanol concentration increased from 10 to $30 \%$.

Effect of ethanol on endotoxin size distribution: Endotoxin size distribution was examined in the presence of different ethanol concentrations of $100 \mathrm{EU} / \mathrm{mL}$ water solution. The results are shown in Fig. 7. In contrast to ethanol-free solutions, marked differences were observed as a function of the solvent condition employed. The endotoxin size distribution was generally largest in ethanol-free solutions. As ethanol concentration increased from 10 to $30 \%$, the endotoxin size distribution decreased from 537.6 to $386.7 \mathrm{~nm}$. These results suggest that the endotoxin removal efficiency increased as endotoxin size distribution increased.

In this study, the endotoxin size distribution increased as temperature rose because of the change in endotoxin agglomerate state. As the temperature increased, the movement rate of endotoxin increased, and the hydration layer between the endotoxin molecules weakened. This change contributed to the agglomeration between endotoxin molecules. This effect led to an increase in endotoxin removal efficiency. The results confirm the positive correlation between endotoxin aggregates and temperature.

When $\mathrm{pH}$ was increased from 3 to 10 , the endotoxin particle size decreased, and endotoxin removal efficiency significantly was reduced from 50 to $20 \%$ by $50 \mathrm{kDa}$ membrane and 28 to $8.3 \%$ by $100 \mathrm{kDa}$ membrane, respectively. These behaviors occur because endotoxin possesses surfactant properties, and its structure has a phosphate radical. As $\mathrm{pH}$ increased, the aggregated endotoxin was dispersed by the Coulomb repulsion of like-charge elements. Therefore, endotoxin existed in monomolecular and low molecular aggregate states. When ethanol was added into the endotoxin aqueous solution, the surface tension of the aqueous solution decreased. As the ethanol concentration increased, the endotoxin surface energy (\%) in alcohol solution was less than that in the aqueous solution. Thus, endotoxin molecules tend to exist in monomolecular and low molecular aggregate states. In addition, endotoxin particle size and endotoxin removal efficiency decreased with increasing ethanol concentration.

\section{ACKNOWLEDGEMENTS}

This work was supported by the National First-Class Key Discipline for Traditional Chinese Medicine of Nanjing University of Chinese Medicine of China (Project No. 2011ZYX3-008) and the State Key Laboratory of New-tech for Chinese Medicine Pharmaceutical Process of China (Project No. SKL2010Z0201).

\section{REFERENCES}

1. A. Billiau and F. Vandekerckhove, Eur. J. Clin. Invest., 21, 559 (1991).

2. K.C. Hou and R. Zaniewski, Biotechnol. Appl. Biochem., 12, 315 (1990).

3. C.B. Weber, B. Henne, F. Loth, M. Schoenhofen and D. Falkenhagen, ASAIO J., 41, M430 (1996).

4. M. Belanich, B. Cummings, D. Grob, J. Klein, A. O'Connor and D. Yarosh, Pharm. Tech., 20, 142 (1996).

5. L. Li and R.G. Luo, Biotechnol. Tech., 12, 119 (1998).

6. L. Li and R.G. Luo, Sep. Sci. Technol., 34, 1729 (1999).

7. Y. Aida and M.J. Pabst, J. Immunol. Methods, 132, 191 (1990).

8. C.Y. Li, Y.F. Zheng, H.Y. Li; Z. Yin and G.P. Peng, Herald of Medicine, 29, 142 (2010).

9. D. Petsch, T.C. Beeskow, F.B. Anspach and W.-D. Deckwer, J. Chromatogr. B, 693, 79 (1997).

10. Y. Kang and R.G. Luo, Process Biochem., 36, 85 (2000).

11. D. Petsch, W.-D. Deckwer, F.B. Anspach, C. Legallais and M. Vijayalakshmi, J. Chromatogr. B, 707, 121 (1998).

12. Y. Kang and R.G. Luo, Am. Biotechnol. Lab., 17, 30 (1999). 